\title{
Tolerogenic dendritic cells in experimental autoimmune encephalomyelitis, specific tolerance induction?
}

\author{
Carla Sellés-Moreno ${ }^{1,2,4^{*}}$, Cristina Ramo-Tello², Laia Grau-López² ${ }^{2}$ Ricardo Pujol-Borrel ${ }^{3,4}$, Eva M Martínez-Cáceres ${ }^{1,4}$ \\ From 7th European Workshop on Immune-Mediated Inflammatory Diseases \\ Noordwijk aan Zee, the Netherlands. 28-30 November 2012
}

\section{Background}

Specific cell therapy with tolerogenic Dendritic Cells (tolDCs) loaded with autoantigens is a promising tool for the attenuation of pathogenic $\mathrm{T}$ cells in autoimmune diseases such as Multiple Sclerosis (MS).

The aim of this study was to analyse the in vitro effect of tolerogenic bone marrow derived DCs (BM-DCs) loaded with Myelin Oligodendrocyte Glycoprotein (MOG) 40-55 peptide from C57BL/6 mice, on the specific proliferation of splenocytes of mice with EAE.

\section{Methods}

Chronic EAE was induced in C57BL/6 mice by s.c. immunization with MOG40-55 emulsified in complete Freund's adjuvant. Pertussis Toxin was injected i.v. at days 0 and 2 post-immunization to increase blood-brain-barrier permeability. Clinical score was daily measured based on tail/leg paralysis.

TolBM-DCs were in vitro differenciated with GM-CSF in the presence of vitamin D3 (VD3) for 8 days.

On day 7, maturation was induced with LPS. Viability, efficiency of differentiation and phenotype of tolBM-DCs were evaluated. To assess antigen specificity, tolBM-DCs were pulsed with MOG40-55, after the maturation stimulus (day 7) for $18 \mathrm{~h}$ and cocultured with syngeneic splenocytes from mice with established EAE.

\section{Results}

TolBM-DCs displayed a semi-mature phenotype, exhibited by low levels of MHC class II and coestimulatory molecules (CD40, CD86) compared to control mature BM-DCs (mBM-DCs) differenciated in the absence of
VD3. MOG40-55 loaded TolDCs showed to be poor stimulators of specific $\mathrm{T}$ cells of mice with $\mathrm{EAE}$, compared to mBM-DCs.

\section{Conclusion}

These results suggest that MOG-loaded TolDCs may be a powerful tool to induce specific $\mathrm{T}$-cell hyporesponsiveness in mice with chronic MOG-induced EAE. Hence, treatment with tolDCs loaded with myelin peptides might be a potential therapy for EAE/MS.

\section{Author details}

'Immunobiology Laboratory For Research And Diagnostic Applications (LIRAD-BST), Badalona, Spain. ${ }^{2}$ Multiple Sclerosis Unit, Dept. of Neurosciences, Germans Trias i Pujol University Hospital (HUGTiP) and Health Science Research Institute (IGTP), Badalona, Spain. ${ }^{3}$ Dept. of Immunology, Hospital Vall d'Hebron, Barcelona, Spain. ${ }^{4}$ Universitat Autònoma Barcelona, (UAB), Spain.

Published: 28 November 2012

\section{doi:10.1186/1479-5876-10-S3-P27}

Cite this article as: Sellés-Moreno et al.: Tolerogenic dendritic cells in experimental autoimmune encephalomyelitis, specific

tolerance induction? Journal of Translational Medicine 2012 10(Suppl 3): P27. 